\title{
Bleeding anorectal varices: successful treatment with transjugular intrahepatic portosystemic shunting (TIPS)
}

\author{
A C Fantin, G Zala, B Risti, J F Debatin, W Schöpke, C Meyenberger
}

\begin{abstract}
A patient with severe recurrent rectal bleeding from anorectal varices due to portal hypertension because of hepatitis $\mathbf{C}$ virus related liver cirrhosis is presented. As illustrated by the report, it is essential to differentiate bleeding anorectal varices from bleeding haemorrhoids because treatment is different. In our patient, implantation of a transjugular intrahepatic portosystemic shunt (TIPS) led to an impressive regression of the anorectal varices, which could be demonstrated by sigmoidoscopy, endosonography, and magnetic resonance imaging. Recurrent rectal bleeding in a patient with portal hypertension should alert the physician to consider anorectal varices. Endoscopic ultra-sound and magnetic resonance imaging are new and non-invasive modalities for diagnosis and post-treatment control.
\end{abstract}

(Gut 1996; 38: 932-935)

Keywords: portal hypertension, anorectal varices, TIPS, endosonography, magnetic resonance imaging.

Portal hypertension leads to the development of collaterals at sites of portal and systemic venous anastomosis. Oesophageal varices are the most common site of major haemorrhage, but varices in unusual extraoesophageal locations have been reported. ${ }^{1-3}$ Despite the high prevalence of anorectal varices in portal hypertension, serious haemorrhage is quite rare but may be fatal. ${ }^{45}$ Various diagnostic procedures have been used for the investigation of anorectal varices in the past, including flexible sigmoidoscopy, ${ }^{2}$ barium enema, ${ }^{6}$ transhepatic splenoportography, ${ }^{7}$ and endoscopic sonography. ${ }^{26-8}$ Although several techniques for treating acute bleeding like surgical suture, sclerotherapy, rubber banding, and rectal package have been reported, the optimal therapeutic management has not been established. ${ }^{19-11}$

We report a patient with severe recurrent rectal bleeding due to $\mathrm{HCV}$ related liver cirrhosis successfully treated by a transjugular intrahepatic portosystemic shunt (TIPS).

\section{Case report}

A 83 year old female patient with liver cirrhosis was referred from an outside hospital for evaluation and treatment of recurrent life threatening rectal bleedings. She had been well until 1992, when she was admitted to the hospital with ascites. A diagnosis of liver cirrhosis resulting from chronic hepatitis $\mathrm{C}$ was made. Other signs of portal hypertension, including non-bleeding oesophageal varices grade IV and splenomegaly, were present. Diuretics were given and ascites improved. The patient was well for the following two years. Liver function tests remained normal. In 1994 recurrent mild rectal bleeding occurred without a need for transfusions. The diagnosis of first degree haemorrhoids was made proctoscopically and local treatment (topical ointments, suppositories) was started. In late 1994 the bleedings became more severe and the patient was readmitted to the same hospital. Emergency sigmoidoscopy was performed revealing active rectal bleeding resulting from second degree haemorrhoids. Rubber band ligation was applied. Immediately after the treatment life threatening bleeding occurred at the site of band ligation. The rectum was packed with gauze and the patient received six units of packed red blood cells and five units of fresh

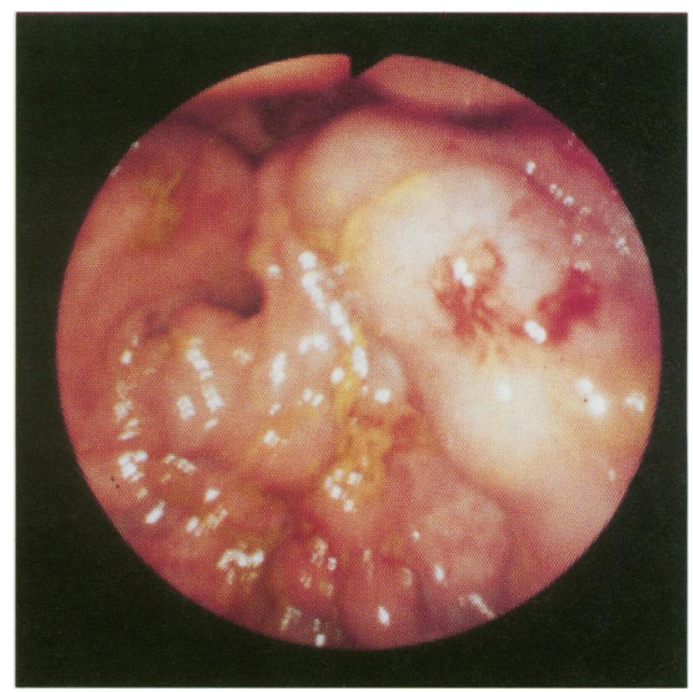

Figure 1: Flexible sigmoidoscopy shows large serpiginous anorectal varices extending from the anal verge to the upper third of the rectum. 
Figure 2: Endosonographic image $(7.5 \mathrm{MHz})$ of a transverse section of the rectum with multiple, large tortuous vascular structures of 7 to $9 \mathrm{~mm}$ in diameter (arrows) in the perirectal space.

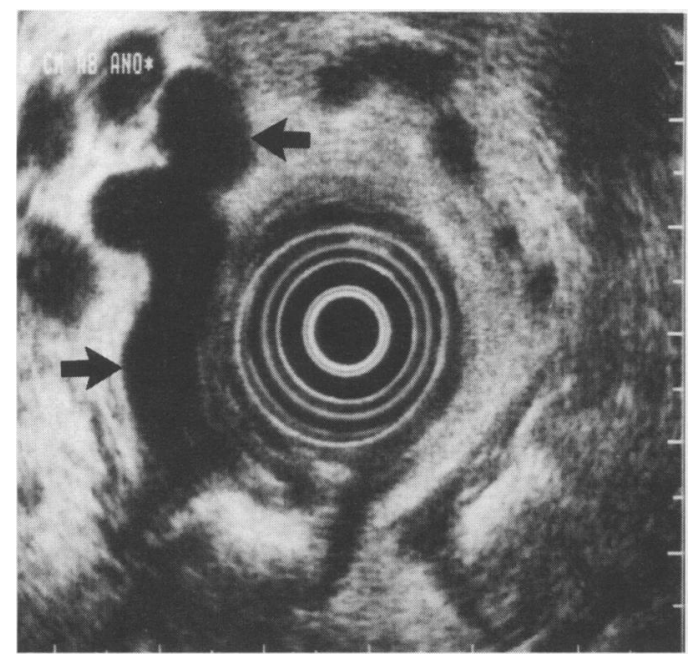

frozen plasma. She was admitted to the intensive care unit and mechanical ventilation was necessary. After recovering within a few days, the patient was transferred to our institution.

On admission physical examination revealed a pale patient with normal vital signs. No hepatomegaly and splenomegaly were seen. Moderate ascites was detectable. Digital rectal examination excluded active bleeding. Laboratory studies were remarkable for the following values: packed cell volume $36 \%$ (NR 37-47), prothrombin time INR $1.6($ NR $0.8-1 \cdot 2)$, alanine transaminase $28 \mathrm{U} / 1$ (NR 3-60), aspartate transferase U/I (10-60), total bilirubin 15 $\mu \mathrm{mol} / 1$ (NR 1-26), albumin $48 \mathrm{~g} / 1$ (NR 34-51). Flexible rectosigmoidoscopy showed second degree haemorrhoids and large serpiginous anorectal varices extending from the anal verge to the upper third of the rectum (Fig 1). Endoscopic ultrasound with a $7.5 \mathrm{MHz}$ radial scanning echoendoscope (GF-UM 20, Olympus Optical, Tokyo, Japan) showed multiple, large tortuous vascular structures of 7 to $9 \mathrm{~mm}$ in diameter in the rectal wall and the perirectal space (Fig 2). The rectal wall was considerably thickened to $24 \mathrm{~mm}$ because of intramural varices (Fig 3).

Magnetic resonance imaging was performed on a 1.5 Tesla MR-system (Signa Advantage, General Electric Medical Systems, Milwaukee, Wisconsin, USA) using the body coil for signal transmission and reception. Axial T1- (TR/TE 400/12) and fast T2- weighted (TR/TE 4000/102) SE images were followed by axial

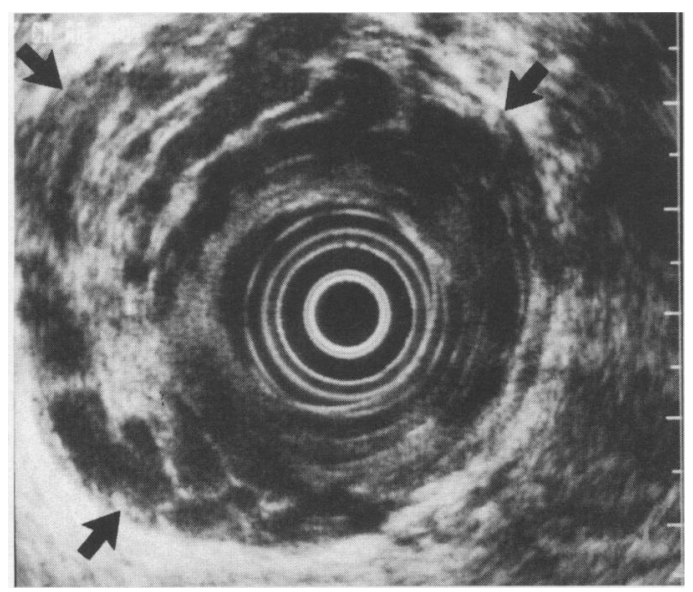

and coronal 'time of flight' gradient echo images (TR/TE/flip $33 / 10 / 30^{\circ}$ ) of the abdominal vasculature. Subsequently, Cine Phase Contrast images (TR/TE/flip 28/8/45, 16 phases/RRinterval, velocity encoding value of $40 \mathrm{~cm} / \mathrm{sec}$ ) were acquired in a plane perpendicular to the course of the portal vein, distal to the portal venous confluens and proximal to the bifurcation, as well as perpendicular to the azygous and haemiazygous veins at the level of T6. The magnetic resonance evaluation showed a cirrhotic liver, splenomegaly, ascites, and moderate gastro-oesophageal varices, resulting in significantly increased flow in the azygous and haemiazygous veins of $404.8 \mathrm{ml} / \mathrm{min}$. Flow in the portal vein was hypodynamic merely amounting to $473.6 \mathrm{ml} / \mathrm{min}$.

Upper gastrointestinal endoscopy showed fourth degree oesophageal varices without red colour signs. Abdominal ultrasound examination showed liver cirrhosis, splenomegaly, and a small amount of ascites. Doppler ultrasound showed a patent portal vein with a mean hepatopetal flow of $17 \mathrm{~cm} / \mathrm{sec}$ (flow volume $473 \mathrm{ml} / \mathrm{min}$ ). For portal decompression a placement of a TIPS seemed to be the most appropriate procedure. Using the right internal jugular vein a $1.8 \mathrm{~mm}$ porto-caval needle (Angiomed, Karlsruhe, BRD) was advanced through a $10 \mathrm{~F}$ introducer sheath under fluoroscopic guidance into the right portal vein close to the portal venous bifurcation. Once the portal vein was entered, a $5 \mathrm{~F}$ catheter was introduced over a 0.035 inch guidewire into the portal vein, respectively the splenic or mesenteric vein. Pressure measurements and a portal venogram were performed to permit calculation of the portosystemic gradient and to identify the hepatofugal flow both in the superior and inferior mesenteric veins, filling the large serpiginous anorectal varices.

A balloon angioplasty catheter, $8 \mathrm{~mm}$ in diameter, was inflated across the parenchymal tract. Subsequently the $10 \mathrm{~F}$ introducer sheath was advanced into the portal vein. Two overlapping balloon expandable Palmaz stents (Johnson and Johnson, Warren, New Jersey, USA) were positioned to bridge the parenchymal tract. The stents were expanded to a

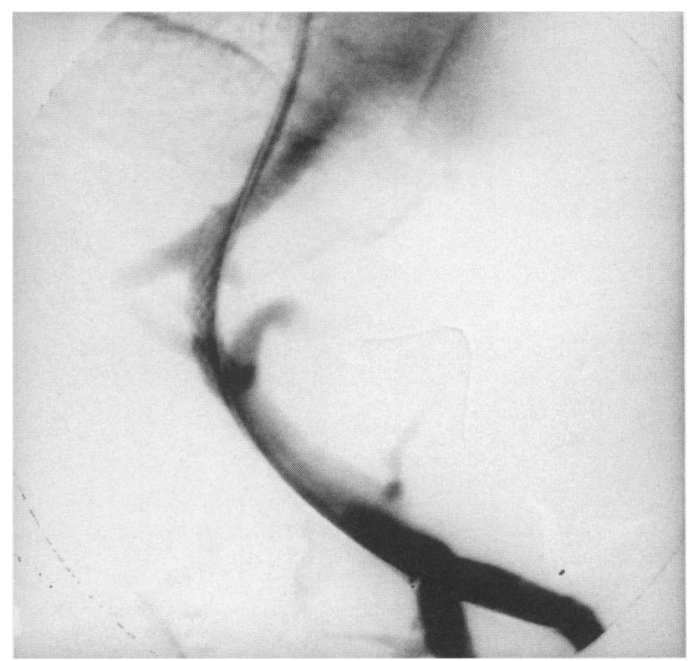

Figure 4: Successful transjugular intrahepatic placement of a self expanding metal stent.
Figure 3: Endosonographic view of the rectum with pronounced wall thickening of $24 \mathrm{~mm}$ because of (arrows). 
Figure 5: Endoscopic ultrasound image with remarkable reduction of the anorectal varices (arrows) after successful TIPS placement.

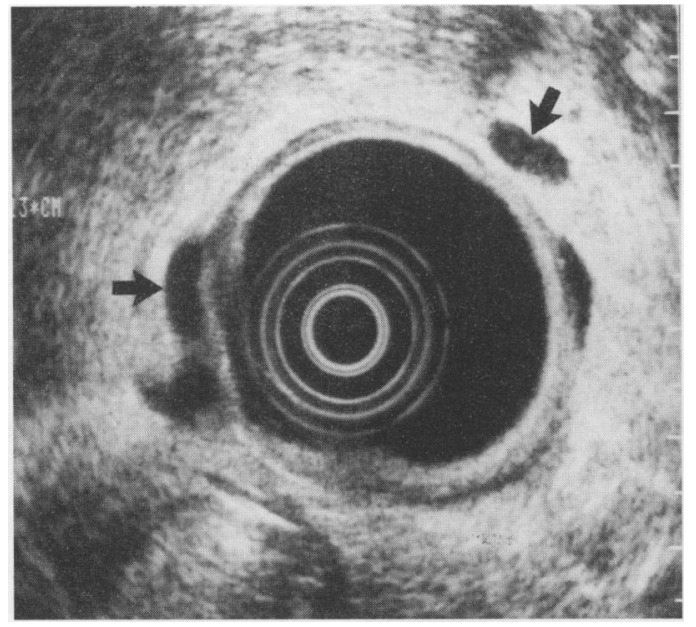

diameter of $9 \mathrm{~mm}$. The portal venogram and pressure measurement were repeated. The pretreatment portosystemic gradient of $29 \mathrm{~mm}$ $\mathrm{Hg}$ could be reduced to $12 \mathrm{~mm} \mathrm{Hg}$ (Fig 4). No procedure related complications occurred. For the next two days the patient received heparin 10000 units per day intravenously followed by low molecular weight heparin 5000 units per day subcutaneously to prevent stent occlusion.

Liver function remained normal and signs of encephalopathy were absent. Repeated endoscopic ultrasound showed remarkable reduction of the anorectal varices (Fig 5) and the wall thickening (Fig 6). Magnetic resonance imaging with portal and azygous venous flow measurement recorded a significant decrease in the mean azygous venous flow from 405 $\mathrm{ml} / \mathrm{min}$ to $161 \mathrm{ml} / \mathrm{min}$ after TIPS placement. The anorectal varices were no longer visible. An impressive regression of the anorectal varices was also confirmed by sigmoidoscopy. Repeated transabdominal sonography with Doppler ultrasound showed a patent stent with hepatopetal flow and an almost complete regression of ascitic fluid. The subsequent course of the patient was uneventful. She was discharged 10 days after admission.

Regular follow up controls (clinical examination, laboratory studies, Duplex-sonographic investigations of stent patency, and function) at two, four, six, eight, 10, and 12 months after discharge confirmed a well patient with functioning TIPS. No further rectal bleeding

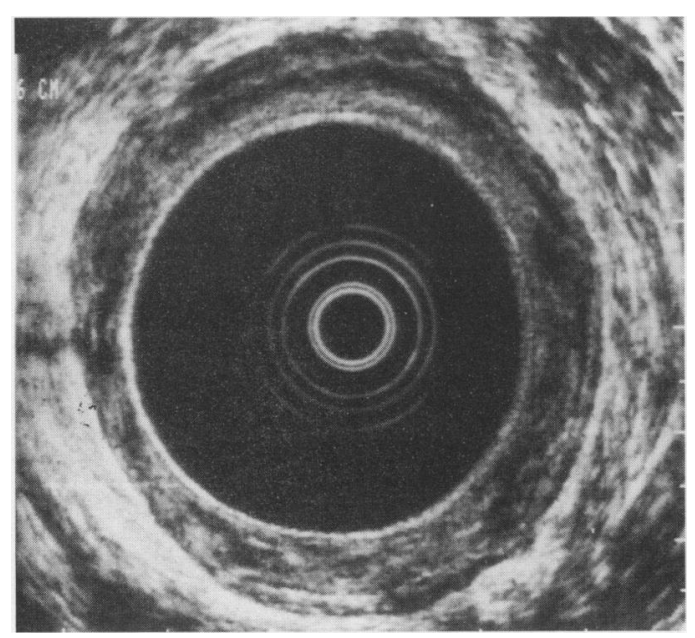

episodes and no signs of hepatic encephalopathy have been noted during this period.

\section{Discussion}

Anorectal varices are an often misdiagnosed condition associated with portal hypertension. As illustrated by our case report, it is essential to differentiate bleeding anorectal varices from bleeding haemorrhoids because treatment is different. ${ }^{41012}$ Haemorrhoids are vascular cushions composed of venular and arteriolar anastomoses without communications to the portal venous system. ${ }^{312}$ At proctoscopy they are purple in colour, usually prolapse into the proctoscope, and do not extend proximal to the denate line. In contrast, anorectal varices are porto-systemic collaterals connecting the superior haemorrhoidal vein (portal system) to the middle and inferior haemorrhoidal vein (systemic circulation). ${ }^{31213}$ They are of dark blue colour, extend from the anal verge to the rectum and do not prolapse into the proctoscope. Haemorrhoids are not more often seen in patients with portal hypertension than in normal persons, ${ }^{411}$ but they can coexist with anorectal varices. Therefore patients with portal hypertension and active rectal bleeding should be examined with flexible sigmoidoscopy, which has been shown to be the most reliable method in diagnosing anorectal varices. ${ }^{2}$ Endoscopic ultrasound, which is now well established for staging rectal cancer, is a new technique permitting excellent visualisation of the rectal wall and the perirectal space, as shown in our patient. ${ }^{8}$ Newer devices combining endoscopic ultrasound with colour Doppler improve the diagnostic capacity further. ${ }^{814}$ The diagnosis of anorectal varices can even be made during active bleeding, which makes sigmoidoscopy difficult. Like other authors we recommend this non-invasive and rapid procedure for diagnosis and post-treatment control of anorectal varices. ${ }^{15}$ Magnetic resonance imaging, a new diagnostic tool in portal hypertension clearly delineates unusual sites of collaterals and provides important information on portal and azygos venous flow. In a study performed at our institution we could demonstrate a highly increased azygos venous flow in portal hypertension. ${ }^{16}$ Furthermore, magnetic resonance imaging is a useful non-invasive method to assess the portal venous system after TIPS placement. ${ }^{5}$ These new techniques can replace the more invasive methods for visualisation of anorectal varices. ${ }^{7} 17$

Reported prevalence of anorectal varices in portal hypertension varies. ${ }^{18}$ Hosking et al ${ }^{13}$ observed anorectal varices in $44 \%$ of prospectively studied cirrhotic patients, whereas Chawla and Dilawari ${ }^{18}$ found an overall frequency of $78 \%$. A significantly higher rate was found in patients with non-cirrhotic portal hypertension $(89 \%)$ compared with patients with liver cirrhosis $(56 \%)$. This is in contrast with the report of Goenka et al, ${ }^{3}$ which did not find any correlation between prevalence of anorectal varices and underlying causes of portal hypertension. While oesophageal varices are known for frequent bleeding, anorectal varices 
have a much lower complication rate. Bleeding rates in patients with confirmed anorectal varices are reported to range from 0.5 to $5 \% .^{5}$ The cause of bleeding remains unknown. ${ }^{1}$ There are only few data of portosystemic pressure gradients in patients with bleeding anorectal varices. In our patient the pretreatment portosystemic gradient of $29 \mathrm{~mm} \mathrm{Hg}$ was considerably increased as shown by Weinshel ${ }^{11}$ and Hsieh. ${ }^{7}$

The optimal therapeutic strategy for bleeding anorectal varices has not yet been established. ${ }^{2}$ Successful control of massive active bleeding might be achieved with sclerotherapy, although fatal outcome has been reported. ${ }^{34}$ Other reported successful treatments in the emergency setting include rubber banding, gel foam embolisation, and surgical suture ligation. ${ }^{10-12}$ Therapeutic failures have been reported for rectal tamponade, ${ }^{24}$ balloon catheters, ${ }^{1}$ Linton tube, ${ }^{19}$ and vasopressin infusions. ${ }^{4}$ However, anorectal varices seem to rebleed in most patients unless a definite reduction of the portal venous pressure is achieved. Intrahepatic portosystemic shunting offers an effective alternative to the surgical approach, as operative shunting carries a considerable morbidity and mortality from procedure related bleeding, hepatic failure, and sepsis. ${ }^{20}$ In $1993 \mathrm{Katz}$ et al ${ }^{5}$ published a report on the first patient who was successfully treated with transjugular intrahepatic portosystemic shunting for recurrent bleeding of anorectal varices despite previous rubber band ligation. After the first studies with humans of Colapinto in $1982,{ }^{21}$ the development of self expanding metal stents made the application for portal decompression possible. TIPS has proved to be an effective treatment to control acute and chronic bleeding of oesophageal varices. ${ }^{22} 23$ As shown by other reports and our own, TIPS placement is successful for the treatment of varices at unusual sites. ${ }^{24}$

Even in an emergency setting the procedure related morbidity and mortality is low, as shown by Rössle et al. ${ }^{23}$ The rebleeding rate for oesophageal varices ranges from 10 to $15 \%$. Although longterm experience of patients with TIPS is limited, we clearly advocate portosystemic shunting as the first therapeutic option in patients with portal hypertension and bleeding anorectal varices. Compared with local treatment including sclerotherapy, rubber banding, and surgical suture, TIPS is a safe and effective alternative therapeutic option with a low complication rate even in older patients, as shown in our report.

After TIPS placement about $20 \%$ to $30 \%$ of patients develop signs of hepatic encephalopathy. ${ }^{25}$ There are only a few reports concerning the risk of hepatic encephalopathy after TIPS procedures in elderly patients. The risk of hepatic encephalopathy after TIPS implantation in patients younger than 60 years is related to the Child-Pugh's class, whereas in older patients (over 60 years) no correlation to the severity of liver cirrhosis could be seen. ${ }^{25}$ Sellinger et $a l^{2627}$ conclude that the risk of hepatic encephalopathy is higher in older patients and that larger stent shunt diameters increase the risk in all patients. They clearly advocate the implantation of smaller $(<8 \mathrm{~mm})$ stents in the high risk population. In our 83 year old patient a $9 \mathrm{~mm}$ diameter stent was implanted. After about 12 months of regular follow up no episodes of hepatic encephalopathy or further rectal bleeding have occurred.

Recurrent rectal bleeding in a patient with portal hypertension should alert the physician to consider anorectal varices. Endoscopic ultrasound and magnetic resonance imaging are new non-invasive imaging modalities for both the diagnosis and post-treatment control in anorectal variceal bleeding.

1 Hosking SW, Johnson AG. Bleeding anorectal varices - a misunderstood condition. Surgery 1988; 104: 70-3.

Herman BE, Baum S, Denobile J, Volpe RJ. Massive bleeding from rectal varices. Am 7 Gastroenterol 1993; 88: 939-42.

3 Goenka MK, Kochhar R, Nagi B, Mehta SK. Rectosigmoid varices and other mucosal changes in patients with portal hypertension. Am $\mathcal{f}$ Gastroenterol 1991; 86: 1185-9.

4 Wang M, Desigan G, Dunn D. Endoscopic sclerotherapy for bleeding rectal varices: a case report. $A m \mathcal{F}$ Gastroenterol 1985; 80: 779-80.

5 Katz JA, Rubin RA, Cope C, Holland G, Brass CA. Recurrent bleeding from anorectal varices: successful treatment with a transjugular portosystemic shunt. $A m \mathcal{F}$ Gastroenterol 1993; 88: 1104-7.

6 Hamlyn AN, Lunzer MR, Morris JS, Puritz H, Dick R. Portal hypertension with varices in unusual sites. Lancet 1974; ii: 1531-4.

7 Hsieh JS, Huang CJ, Huang YS, Huang TJ. Demonstration of rectal varices by transhepatic inferior mesenteric venography. Dis Colon Rectum 1986; 29: 459-61.

8 Lee SH. Case report: Transrectal ultrasound in the diagnosis of anorectal varices. Clin Radiol 1994; 49: 69-70.

9 Richon J, Berclaz R, Schneider PA, Marti MC. Sclerotherapy of rectal varices. Int f Colorect Dis 1988; 3: 132-4.
then J, Berclaz R, Schneider PA, Marti MC. Sclerotherapy of rectal varices. Int $\mathcal{F}$ Colorect Dis 1988; 3: 132-4.
10 Keane RM, Britton DC. Massive bleeding from rectal varices following repeated injection sclerotherapy of oesophageal varices. Br f Surg 1986; 73: 120.

11 Weinshel E, Chen W, Falkenstein DB, Kessler R, Raicht RF. Hemorrhoids or rectal varices: defining the cause of massive rectal hemmorhage in patients with portal hypertension. Gastroenterology 1986; 90: 744-7.

12 McCormack TT, Bailey HR, Simms JM, Johnson AG.

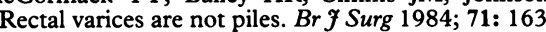

13 Hosking SW, Johnson AG, Smart HL, Triger DR. Anorectal varices, hemorrhoids and portal hypertension. Lancet 1989; i: 349-52.

14 Giovannini M, Seitz JF. Endoscopic ultrasonography with a linear-type echoendoscope in the evaluation of 94 patients linear-type echoendoscope in the evaluation of 94 patients

15 Killi MR, Ozutemiz O, Sevinc E. Transrectal ultrasonographic demonstration of rectal varices [letter]. $A m \mathcal{F}$ Gastroenterol 1992; 87: 1685-6.

16 Debatin JF, Zahner B, Meyenberger C, Romanowski B, Schöpke W, Marincek B. Cine-PC quantitation of azygos blood flow in volunteers and patients with portal hypertension before and after TIPS. Hepatology (in press).

17 Waxman JS, Tarkin N, Dave P, Waxman M. Fatal hemorrhage from rectal varices. Report of two cases. Dis Colon Rectum 1984; 27: 749-50.

18 Chawla YK, Dilawari JB. Anorectal varices haemorrhoids and portal hypertension [letter]. Lancet 1989; i: 725.

19 Weiserbs DB, Zfass AM, Messmer J. Control of massive hemorrhage from rectal varices with sclerotherapy. Gastrointest Endosc 1986; 32: 419-21.

20 Jin G, Rikkers LF. Cause and management of upper gastrointestinal bleeding after distal splenorenal shunt. Surgery 1992; 112: 719-27.

21 Colapinto RF, Stonell RD, Birch SJ. Creation of an intrahepatic portosystemic shunt with a Gruntzig balloon catheter. Can Med Assoc F 1982; 126: 267-8.

22 La Berge JM, Ring EJ, Gordon RL, Lake JR, Doherty MM, Gomberg KJ, et al. Creation of transjugular intrahepatic portosystemic shunts with the wallstent endoprosthesis: results in 100 patients. Radiology 1993; 187: 413-20.

23 Rössle M, Haag K, Ochs A, Sellinger M, Nöldge G, Perarnau J, et al. The transjugular intrahepatic portosystemic stent-shunt procedure for variceal bleeding. $N$ Engl temic stent-shunt procedure

24 Harrison CA, Benner KG, Sahagun G. Ectopic variceal hemorrhage managed by TIPS. Hepatology 1992; 116: 304-9.

25 Haag K, Ochs A. Transjugular intrahepatic portosystemic stent-shunt in the treatment of portal hypertension. Current Opinion in Gastroenterology 1993; 9: 435-40.

26 Sellinger M, Haag K, Ochs A, Noeldge G, Gerok W, Rössle $M$. Factors influencing the incidence of hepatic encephalopathy in patients with transjugular intrahepatic portosystemic stent-shunt (TIPS). Hepatology 1992; 16: 122A

27 Sellinger M, Ochs A, Haag K, Rössle M. Hepatic encephalopathy in patients with transjugular intrahepatic portosystemic stent-shunt (TIPS). $\mathcal{J}$ Hepatol 1993; 18 (suppl 1): S15. 